\title{
TU/e EmonOWEN

\section{Efficient modeling of multistage integrated circuit passive isolation structures}

\section{Citation for published version (APA):}

Grau Novellas, M., Serra, R., Rose, M., \& Secareanu, R. (2018). Efficient modeling of multistage integrated circuit passive isolation structures. IEEE Transactions on Electromagnetic Compatibility, 60(2), 544 - 547 . [7953570]. https://doi.org/10.1109/TEMC.2017.2714859

DOI:

10.1109/TEMC.2017.2714859

Document status and date:

Published: 01/04/2018

\section{Document Version:}

Accepted manuscript including changes made at the peer-review stage

\section{Please check the document version of this publication:}

- A submitted manuscript is the version of the article upon submission and before peer-review. There can be important differences between the submitted version and the official published version of record. People interested in the research are advised to contact the author for the final version of the publication, or visit the $\mathrm{DOI}$ to the publisher's website.

- The final author version and the galley proof are versions of the publication after peer review.

- The final published version features the final layout of the paper including the volume, issue and page numbers.

Link to publication

\section{General rights}

Copyright and moral rights for the publications made accessible in the public portal are retained by the authors and/or other copyright owners and it is a condition of accessing publications that users recognise and abide by the legal requirements associated with these rights.

- Users may download and print one copy of any publication from the public portal for the purpose of private study or research.

- You may not further distribute the material or use it for any profit-making activity or commercial gain

- You may freely distribute the URL identifying the publication in the public portal.

If the publication is distributed under the terms of Article 25fa of the Dutch Copyright Act, indicated by the "Taverne" license above, please follow below link for the End User Agreement:

www.tue.nl/taverne

Take down policy

If you believe that this document breaches copyright please contact us at:

openaccess@tue.nl

providing details and we will investigate your claim. 


\title{
Efficient Modeling of Multi-Stage Integrated Circuit Passive Isolation Structures
}

\author{
Mercè Grau Novellas, Ramiro Serra, Matthias Rose and Radu Secareanu
}

\begin{abstract}
In this paper, an efficient electromagnetic modeling methodology is proposed for multi-stage integrated passive isolation structures. The methodology is based on a modal expansion of interference field components. With it, we are able to accurately characterize a great variety of structures approximately 300 times faster than with full-wave simulations. Calculated results have been compared to both simulations and measurements, obtaining good agreement. The proposed model is intended to be a tool for fast prediction purposes at early stages of the design flow.
\end{abstract}

Index Terms-Electromagnetic interference, integrated circuits, signal integrity, substrate coupling.

\section{INTRODUCTION}

$\mathbf{I}$ $\mathrm{N}$ the past years, integration levels in electronic systems have been continuously increasing so as to enable the development of high-performance products and applications [1]. This implies that different electronic functional blocks (e.g. ADC's, RF modules, etc) are implemented in the same substrate and, therefore, share a common path for potentially unwanted coupling between them. In this context, to characterize and provide insight on electromagnetic interference propagation mechanisms in multilayered substrates is a key point to enable a disturbance-aware design environment and, consequently, to avoid expensive design iterations.

The continuous increase of operating frequencies pushes the validity limit of static or quasi-static approximations. An investigation of their validity in the context of integrated circuits (IC) substrate modeling can be found in [2]. In addition, it also pushes the bandwidth of validity of behavioral equivalent RC networks, which are based on single-mode representations, as they neglect the effects derived from multimodal propagation [3] . Consequently, there is a need for models able to account for electromagnetic effects [4], in an efficient way.

In order to prevent two sections of the same IC from interfering each other, highly resistive and/or charge-free sections are commonly embedded in order to increase isolation between them [5]. Their role is to discontinue the substrate top layer (active layer), where circuits are implemented, and thus interrupt the normal flow of currents that support interference propagation. Consequently, this type of structures can be considered as discontinuities transverse to the direction of interference propagation. Therefore, they can cause effects

This research project has been supported by a STW-HTSM Program of The Netherlands, project number 12853 .

M. Grau Novellas and R. Serra are with the Department of Electrical Engineering, Eindhoven University of Technology, 5600MB Eindhoven, The Netherlands (e-mail: m.grau@tue.nl).

M. Rose is with NXP Semiconductors, 5656AE Eindhoven, Netherlands.

$\mathrm{R}$. Secareanu is with NXP Semiconductors, Tempe, Arizona. such as mode conversion and mode enhancement, which can modify interference propagation patterns, as described in [6].

In the present paper, a novel modeling methodology is proposed to characterize interference propagation in multilayered substrates in the presence of multi-stage discontinuities. This methodology builds up on previous work [6], where a single stage discontinuity model was proposed that was able to efficiently characterize the effect of $\mathrm{SiO}_{2}$ trenches on interference propagation, providing insight on the isolation effectiveness of such structures. In the present work, the concept is applied to multi-stage discontinuities, in order to model complex passive isolation structures of different kinds (e.g. triple wells or filled trenches) or multiple cascaded structures. The developed methodology efficiently deals with multiple sections that are not bounded by conductive layers, which is the common case for ICs [3]. The proposed model is intended to be a tool for fast interference prediction purposes, that provides insight on the effect of technological boundaries in interference propagation and effectiveness of isolation structures.

\section{Description of the Structures Under Study}

IC substrates can be modeled as a multilayered stack, where the cross section doping profile is decomposed in $m$ layers in the vertical direction and can be considered practically homogeneous in the radial direction. We consider that layer $m+1$ is a semi-infinite non-conductive region and that layer 1 is bounded by a perfect electric conductor (PEC) at the bottom. If this is not the case, the ground plane of the printed circuit board can be used as a reference by adding additional layers. Other boundary conditions can be considered by modifying the expressions of fields induced inside the stack [3].

The physical properties of the layer stack determine the propagation modes that the substrate is able to support. As the structure is semi-open, both discrete and continuous modes can propagate. Discrete modes correspond to surface waves, while continuous modes can be either propagative, which account for radiation into the open-space, or evanescent, which account for energy storage close to the source [7].

Considering the standard IC substrates [3], despite the fact that these structures are open, there is usually an intermediate and/or top layer (active layer) with a relatively high conductivity (typical values are between 100 and $2000 \mathrm{~S} / \mathrm{m}$ ). In the presence of these layers, surface waves are dominant among the continuous spectrum close to the source. In [3], a study can be found on the distance range in which propagation can be characterized only with discrete modes, for different standard IC substrates, and for different conductivities and frequencies.

In this context, implemented isolation structures feature one or multiple sections with different doping profiles, embedded 
in the original multilayered substrate. Each of those sections is able to support a different set of modes. Due to the fact that the primary function of these structures is to interrupt the active layer, at least one or several stages do not include intermediate or top conductive layers. Even though they are still able to support propagation of surface waves, the dominant propagation modes close to the source belong to the continuous spectrum for such stages.

Therefore, as shown in Fig. 1, the structures under study will consist of $N$ consecutive sections with different doping profiles. Each section is then characterized by its number of layers $m$ and, subsequently, each layer by its conductivity $\sigma_{n}$, permittivity $\varepsilon_{n}$ and height $h_{n}$. The source of interference will be a certain current density distribution located in section 1, sections 2 to $N-1$ have isolation purposes and section $N$ is where a potential victim is located.

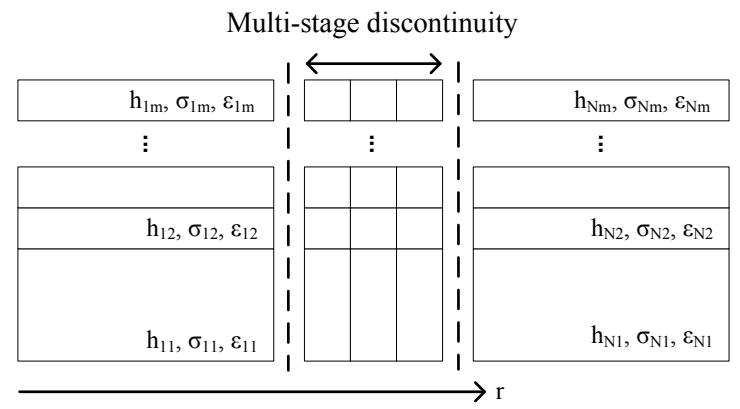

Fig. 1. General diagram of the structures under study.

As interference propagates radially from the source [3], for the sake of simplicity we will consider that the structure described in Fig. 1 is radially symmetric, and thus the different sections have the shape of concentric rings. In this type of structures, interference propagates mainly by means of Transverse Magnetic modes $\left(\mathrm{TM}^{z}\right)$, with field components $E_{z}$, $E_{r}$ and $H_{\phi}$. Sections will be characterized by their absolute radius $\left(r_{1}\right.$ to $\left.r_{N}\right)$.

\section{Multi-Stage Discontinuity Modeling}

When a certain mode, excited in a particular waveguide section, meets a discontinuity, part of the energy is transferred to the subsequent section and part of the energy is reflected. The energy is transferred to all the modes that the new section is able to support and, in the same way, it is reflected to all the modes that the first section is able to support. Using the expressions of the tangential field components at the discontinuity interface together with the orthogonality property of the set of modes in each section, one can write equations to relate the different modal parameters at both sides of the discontinuity. A detailed explanation of the procedure for a 2 -stage discontinuity in closed waveguides can be found in [8].

In the case of having an upper open boundary, the discontinuity interface should be extended to infinity. In addition, the modal expansion of tangential fields should be modified to include the contribution of the continuous spectrum. Thus the general expressions for electric and magnetic field components in a certain section excited with mode 1 will be

$$
\begin{aligned}
& \mathbf{E}=a_{1} \mathbf{e}_{1}+\sum_{i=1}^{\infty} a_{1} \rho_{i_{-} 1} \mathbf{e}_{i}+\int_{\mathcal{D}} a_{1} \rho_{1}\left(k_{r}\right) \mathbf{e}\left(k_{r}\right) \mathrm{d} k_{r}, \\
& \mathbf{H}=a_{1} \mathbf{h}_{1}+\sum_{i=1}^{\infty} a_{1} \rho_{i_{-} 1} \mathbf{h}_{i}+\int_{\mathcal{D}} a_{1} \rho_{1}\left(k_{r}\right) \mathbf{h}\left(k_{r}\right) \mathrm{d} k_{r},
\end{aligned}
$$

where $a_{i}$ are the modal amplitude coefficients, $\rho_{i \_1}$ is the reflection coefficient of mode $i$ when the discontinuity is excited with mode 1 and $\mathcal{D}$ is the integration domain in the complex plane.

Given a certain discontinuity (1 $\mid 2)$ in a multi-stage scenario, where Section 1 is characterized by $p$ modes and Section 2 is characterized by $t$ modes, the inputs of its system of equations will be the amplitude coefficient of the excitation mode (e.g. $a_{11}$ ), and the $t \times t$ reflection coefficients at the subsequent section $\left(\rho_{21} \_2\right.$ to $\left.\rho_{2 t} 2 t\right)$. The corresponding unknowns to be obtained are the $t$ amplitude coefficients at the subsequent section for the given excitation $\left(a_{21} 11\right.$ to $\left.a_{2 t_{-} 11}\right)$ and the $p$ reflection coefficients at the prior section $\left(\rho_{11 \_11}\right.$ to $\left.\rho_{1 p_{-} 11}\right)$. Finally, this system of equations has to be solved $p$ times, for all possible excitations $\left(a_{11}\right.$ to $\left.a_{1 p}\right)$. Consequently, in order to be able to solve the complete structure, the system of equations that corresponds to the discontinuity located further away from the source $(N-1 \mid N)$ has to be solved first [8]. Figure 2 shows the solution flow of the complete set of systems of equations.

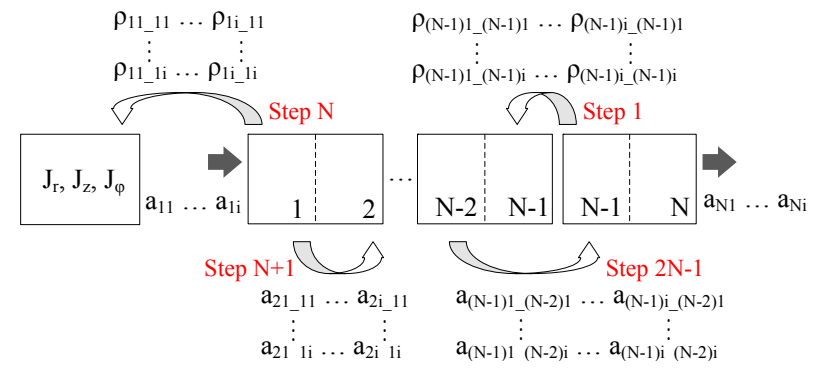

Fig. 2. Solution flow of the complete multi-stage structure, where each box represents a discontinuity interface.

Taking into account the field modal expansion expressions (1)-(2), if a discretization of the continuous spectrum is performed and after obtaining all the intermediate parameters, the amplitude coefficients of interference propagation modes in section $\mathrm{N}$ can be obtained as follows

$$
a_{N i}=\sum_{q_{1}=1}^{\infty} a_{1 q_{1}} \sum_{q_{2}=1}^{\infty} a_{2 q_{2} \_} 1 q_{1} \ldots \sum_{q_{N-1}=1}^{\infty} a_{N i_{-}(N-1) q_{N-1}} .
$$

It is obvious that a truncation of the number of modes considered in each section is needed to be able to solve the problem. In addition, even with a finite number of systems of equations, the complete problem can still be very complex and computationally expensive. For this reason, a simplification is required to be able to characterize propagation through a multi-stage discontinuity in an efficient way.

At this point, we will characterize each section with the few most significant modes, i.e. those with a greater contribution to energy transfer between sections. This means that instead of focusing on accurately describing the fields in the intermediate 


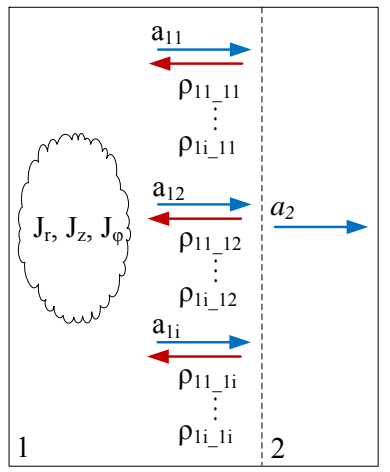

(a)

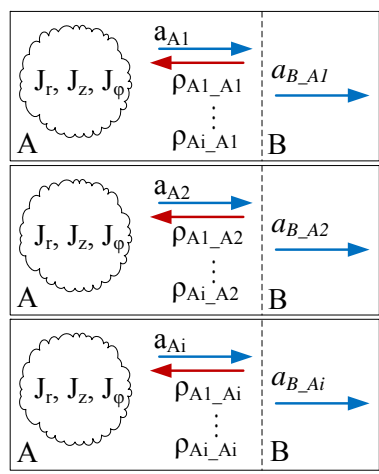

(b)
Fig. 3. Optimization strategies considered: (a)known source and (b) arbitrary source.

sections, we prioritize an accurate description of fields at the first and last sections. Taking into account the main application of the structures under study, this will allow us to obtain an accurate transfer function and therefore apply this methodology for isolation effectiveness studies.

The criteria for choosing the most significant modes is described below. Those sections with top or intermediate relatively highly conductive layers (as described in Sec. II), will be characterized only by the propagating discrete modes. These are obtained by means of a transcendental equation that can be derived when fields are forced to be evanescent in layer $m+1$ [3]. For all other sections, we will consider the discrete propagating modes plus the spectral components that maximize the energy transfer or, in other words, the spectral components that are better matched with the modes exciting the discontinuity interface. In order to optimize the selection of these modes and avoid iterations, instead of taking into account the complete structure, we will just consider the discontinuity to be optimized. The prior section will be fully characterized with all relevant excitation modes and in the following section we will consider only the spectral component to be optimized. At this point, two different strategies can be followed. If the interference source current distribution is known and located in the section before the discontinuity, the amplitude coefficients of each excitation mode are known. Therefore all excitation modes can be considered simultaneously and the spectral component that maximizes the overall transmission can be selected. On the other hand, if the weighted contribution of each excitation mode is not known, an arbitrary source will be used and the matching with each excitation mode will be optimized separately. Figure 3 shows a diagram of both strategies.

Once all the structure parameters are known, we can use different metrics to characterize its isolation. We obtain the transfer function by calculating the ratio of the voltage induced across the stack at the source and victim positions (equivalent to a $S_{21}$ ) and its isolation effectiveness as the ratio of the fundamental mode amplitude coefficient at the victim section, with and without isolation structure. A discussion of those metrics and the effects that they take into account can be found in [6].

\section{EXPERIMENTAL VALIDATION}

In order to assess the validity of the proposed modeling methodology, it will be applied to characterize two types of structures: a triple well and a filled trench. The first one consists of two wells of different doping type (one inside the other) so that, together with the substrate, two consecutive reverse-biased junctions are formed, as sketched in Fig.4-a. The second one consists of a multi-layer stack formed by a p-well, a p-epitaxial layer, a buried oxide and a n-substrate, where a $\mathrm{SiO}_{2}$ trench is embedded and filled with doped polysilicon, so it contacts the substrate, as sketched in Fig.4-b.

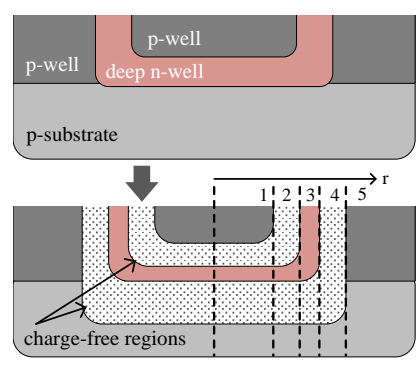

(a)

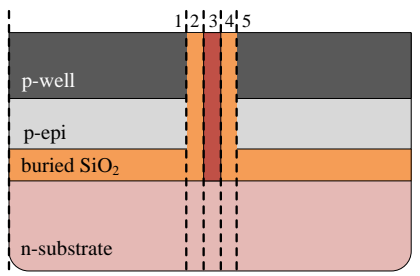

(b)
Fig. 4. Structure of the study cases considered: (a) triple well and (b) filled trench.

The triple well is simulated with the 3D full-wave tool CST Microwave Studio, [9] in order to obtain the fields induced inside the substrate stack. The current density used as a source is a vertically oriented one, flowing across the whole stack height $\mathbf{J}=I \delta(r) \hat{z}$. The structure is modeled with 5 sections of absolute radius $r_{1}=50 \mu \mathrm{m}, r_{2}=50.5 \mu \mathrm{m}, r_{3}=51$ $\mu \mathrm{m}$ and $r_{4}=51.5 \mu \mathrm{m}$. The parallel charge-free regions are 1 $\mu \mathrm{m}$ and $0.5 \mu \mathrm{m}$ thick respectively. The substrate considered is highly resistive $(\sigma=10 \mathrm{~S} / \mathrm{m})$ and $250 \mu \mathrm{m}$ thick. The active layer has a conductivity of $1000 \mathrm{~S} / \mathrm{m}$ and a thickness of $1.5 \mu \mathrm{m}$. The conductivity of the wells is $1000 \mathrm{~S} / \mathrm{m}$ for the $n$ case and $1500 \mathrm{~S} / \mathrm{m}$ for the $\mathrm{p}$ case. Section 4 is the only one without intermediate or top conductive layers, so in this case an optimization is carried out with an arbitrary source. Propagation coefficients are obtained in each section, in the range from $1 \mathrm{MHz}$ to $10 \mathrm{GHz}$ [3]. According to the criteria described in Sec. III, either two or three modes are used to characterize each section. Subsequently, the procedure described in Fig. 2 is applied to obtain all coefficients. The structure has been simulated using a frequency domain solver and was excited with a current port. The total computation time was 12 minutes for 100 frequency points, while simulation time in the same computer was three hours for five frequency points (300 times faster for each frequency point).

There is good agreement between the simulated and the calculated results (see Fig. 5). After the transverse discontinuities location, one can observe a transitory decrease of the field magnitude. This is due to the fact that the high-order modes that are excited interact destructively with the fundamental propagation mode in section 5. However, they attenuate faster and after a certain distance this effect is not present anymore. The simulated and calculated transitory magnitude drops do not coincide. However, this phenomena can be more accurately 

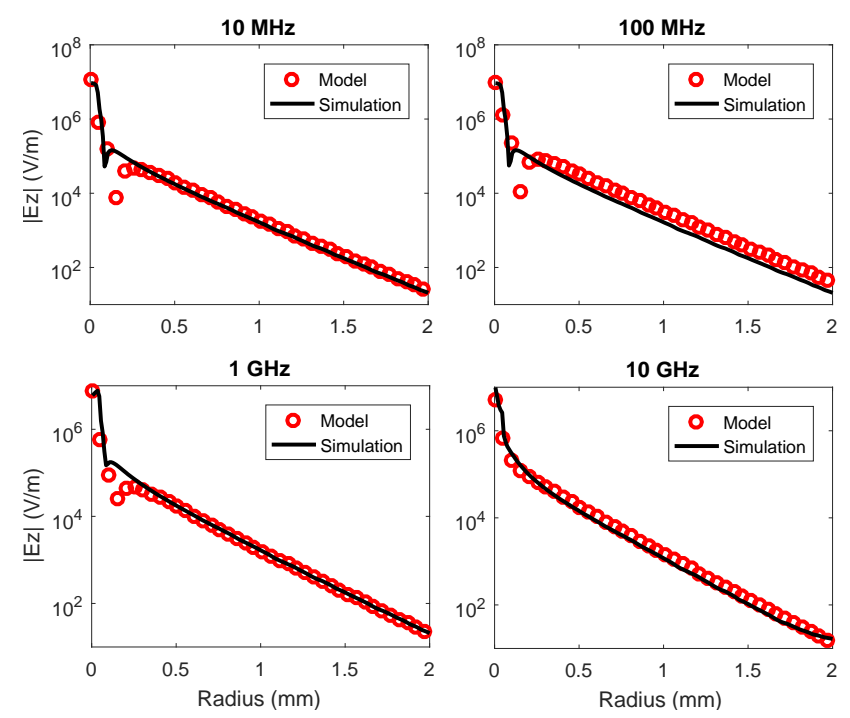

Fig. 5. Magnitude of the $E_{z}$ component induced in layer 1 in the triple well structure at $z=240 \mu \mathrm{m}$, where the effect of mode interaction is higher.

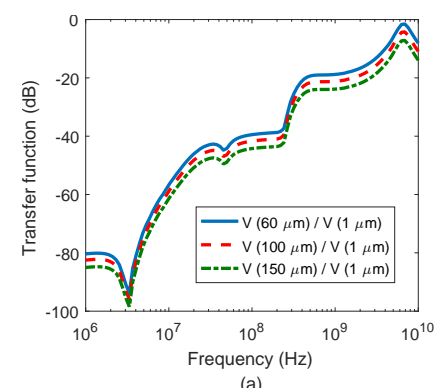

(a)

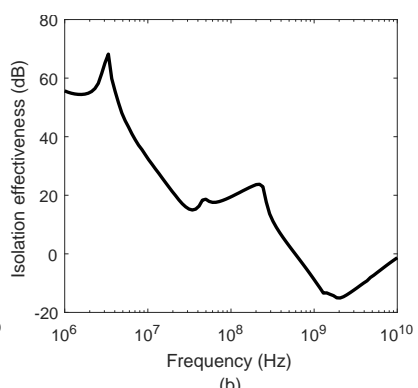

(b)
Fig. 6. Characterization of the triple well structure: (a) voltage ratio and (b) isolation effectiveness.

characterized if additional evanescent modes are included in this section, at the obvious expense of increasing complexity. In Fig. 6 we show the calculated transfer function and isolation effectiveness.

In order to characterize the filled trench case, we have implemented a test structure in a $0.13 \mu \mathrm{m}$ SOI technology that consists of two squared p-well tubs of $200 \times 200 \mu \mathrm{m}$ separated by deep trench isolation (DTI) (see Fig. 4b). In order to obtain the transfer function, it has been embedded in a coplanar ground-signal-ground structure. The layout is shown in Fig. 7. The measured $S_{21}$ is compared to a calculated transfer function obtained from a radially symmetric version of the structure. Results are shown in Fig. 8.

As it can be seen, there is quite good agreement between the measured and calculated results, except from the range between $100 \mathrm{kHz}$ and $1 \mathrm{MHz}$, where measured results reached

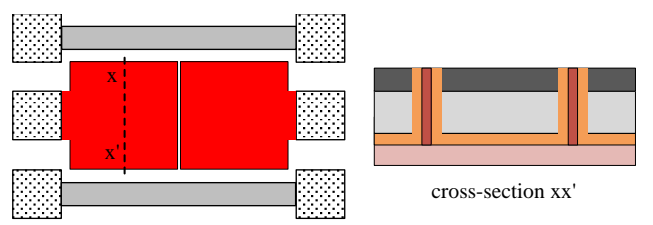

Fig. 7. Layout of the implemented test structure.

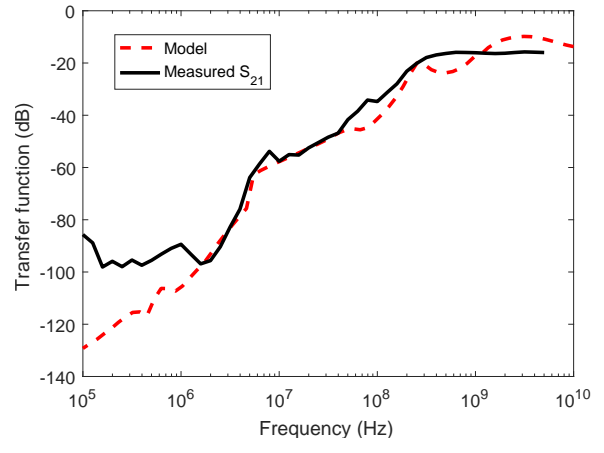

Fig. 8. Comparison of the measured and calculated DTI transfer function.

noise floor. Even though the shape of the test structure is not radially symmetric, the results obtained with the proposed model show that the behavior can be extrapolated, providing a reasonable accurate transfer function. In this case, computation time was eight minutes for 100 frequency points.

\section{CONCLUSION}

This paper presents a modeling methodology for efficient electromagnetic characterization of multi-stage passive isolation structures embedded in IC substrates. We have shown that with a smart selection of the most representative modes, the proposed model is able to accurately represent structures formed by multiple sections of different doping profiles in an efficient way. We have compared the calculated results for triple wells and filled trenches to full-wave simulations and measurements, respectively, obtaining very good agreement. The proposed formulation has been developed assuming radially symmetric structures. The comparison to measurements has shown that, even if the structure characterized experimentally was not radially symmetric, the calculated results can still be extrapolated to other topologies.

\section{REFERENCES}

[1] M. Badaroglu, P. Wambacq, G. V. der Plas, S. Donnay, G. G. E. Gielen, and H. J. D. Man, "Evolution of substrate noise generation mechanisms with CMOS technology scaling," IEEE Transactions on Circuits and Systems, vol. 53, no. 2, pp. 296-305, 2006.

[2] G. Manetas, V. Kourkoulos, and A. Cangellaris, "Investigation on the frequency range of validity of electroquasistatic RC models for semiconductor substrate coupling modeling," IEEE Transactions on Electromagnetic Compatibility, vol. 49, no. 3, pp. 577-584, 2007.

[3] M. Grau-Novellas, R. Serra, and M. Rose, "Methodology for coupling and interference prediction in integrated circuit substrates," IEEE Transactions on Electromagnetic Compatibility, vol. 58, no. 4, pp. 1118 - 1127, 2016.

[4] X. Aragones and A. Rubio, "Challenges for signal integrity prediction in the next decade," Materials Science in Semiconductor Processing, vol. 6, pp. 107-117, 2003.

[5] S. Bronckers, G. V. der Plas, G. Vandersteen, and Y. Rolain., Substrate Noise Coupling on Analog/RF Circuits, 1st ed. Massachusetts: Artech House, 2010.

[6] M.Grau-Novellas, R. Serra, and M. Rose, "Modeling of trench structures in integrated circuits for fast isolation effectiveness assessment," Proc. International Symposium in Electromagnetic Compatibility - EMC Europe, pp. 536-540, September, 2016.

[7] A. W. Snyder and J. D. Love, Optical Waveguide Theory, 1st ed. Boston, MA: Springer, 1983.

[8] A. Wexler, "Solution of waveguide discontinuities by modal analysis," IEEE Transactions on Microwave Theory and Techniques, vol. 15, no. 9, pp. 508-517, 1967.

[9] CST, "CST Microwave Studio - Computer Simulation Technology, 06.2014, https://www.cst.com." 